\title{
t tbar + isolated photon production at NLO accuracy matched with parton shower
}

\section{Zoltán TRÓCSÁNYI*†}

Institute of Physics, University of Debrecen, H-4010 Debrecen P.O.Box 105, Hungary

E-mail: zoltan.trocsanyi@cern.ch

\section{Adam KARDOS}

MTA-DE Particle Physics Research Group, Debrecen, Hungary

E-mail: kardos.adamescience.unideb.hu

\begin{abstract}
We simulate the hadroproduction of a $\mathrm{t} \overline{\mathrm{t}}$-pair in association with a hard photon at LHC using the PowHel package. The generated events are stored according to the Les-Houches event format and constitute an almost inclusive event sample (regarding the photons), so that usual experimental photon isolation can be employed. We interface those events to the PYTHIA shower Monte Carlo program, allowing for decays of massive particles, showering and hadronization, and present predictions for differential distributions at the hadron level.
\end{abstract}

The European Physical Society Conference on High Energy Physics 22-29 July, 2015

Vienna, Austria

\footnotetext{
* Speaker.

$\dagger$ This research was supported by the Swiss National Science Foundation (grant SCOPES IZ73Z0_152601), the Hungarian Scientific Research Fund grant K-101482, the Post Doctoral Fellowship programme of the Hungarian Academy of Sciences and the Research Funding Program ARISTEIA, HOCTools (co-financed by the European Union (European Social Fund ESF) and Greek national funds through the Operational Program "Education and Lifelong Learning" of the National Strategic Reference Framework (NSRF)).
} 
After the discovery of a Higgs-particle at the LHC [1,2], measuring its couplings to the particles of the standard model is a prime task for the experiments. In this respect, the t-quark with mass larger than that of the Higgs-boson has a special status because the latter cannot decay into a t $\bar{t}$-pair. Thus to measure the $\mathrm{t} \overline{\mathrm{t}} H$ coupling in a model independent way, one should study the hadroproduction of the Higgs-boson in association with a ț pair [4, 5]. Measuring this coupling directly is important also because deviation from the SM expectation could provide signal of physics beyond the SM.

Measuring the $\mathrm{t} \overline{\mathrm{t}} H$ production cross section is very challenging for two reasons: the small production rates are often accompanied with large backgrounds. The experiments at the LHC study many decay channels sorted into three main categories: (i) the hadronic, (ii) the leptonic and the (iii) di-photon channels. In this talk we concentrate on the QCD background to the di-photon channel where the Higgs-boson decays into a photon pair, while the t-quarks decay into jets (di-photon with hadrons), or into the semileptonic channel (di-photon with lepton). The irreducible QCD background contains two hard photons in association with a t⿱t -pair. In order to be well detected, the photons have to be isolated.

Isolated hard photons are important tools of experimental particle physics. From the theoretical point of view however, these are rather cumbersome objects. The photons couple directly to quarks. The emission of a photon off a light quark, treated massless in perturbative QCD, is enhanced at small angles and singular for strictly collinear emission. This singularity can be factorized into the photon fragmentation function [6] in a similar way as the initial state collinear singularities can be factorized into parton distribution functions. This way the theoretical prediction can be written as a sum of the perturbatively computable direct photon contribution and the non-perturbative fragmentation term. The fragmentation functions have to be extracted from comparison to measured cross sections, just like the parton distribution functions. The important difference is that in the measured data the fragmentation components are suppressed by the isolation of the photon, therefore the accuracy of this extraction is limited by both statistics and systematics.

It is also possible to define a photon isolation that avoids the fragmentation contribution completely [7], called smooth isolation. The smooth isolation allows QCD activity in a continuously shrinking cone around the photon such that the allowed activity decreases with decreasing cone size according to the formula

$$
E_{\perp, \text { had }}=\sum_{i \in \text { partons }} E_{\perp, i} \Theta\left(\delta-R\left(p_{\gamma}, p_{i}\right)\right) \leq E_{\perp, \gamma}\left(\frac{1-\cos \delta}{1-\cos \delta_{0}}\right),
$$

for all $\delta \leq \delta_{0}$, where $\delta_{0}$ is a sufficiently small, pre-defined number. (For simplicity, we set $\varepsilon_{\gamma}=$ $n=1$ of the original definition in Ref. [7].) This theoretically preferred isolation is in contrast with the usual experimental definition of an isolated photon which allows small hadronic activity inside the isolation cone as follows:

$$
E_{\perp, \text { had }}=\sum_{i \in \text { tracks }} E_{\perp, i} \Theta\left(R_{\gamma}-R\left(p_{\gamma}, p_{i}\right)\right)<E_{\perp, \text { had }}^{\max } .
$$

In Eqs. (1) and (2) $E_{\perp, i}$ is the transverse energy of the $i$ th track, $R_{\gamma}$ is the isolation cone size, $R\left(p_{\gamma}, p_{i}\right)$ is the separation between the photon and the $i$ th track measured in rapidity-azimuthal angle plane, while $E_{\text {had }}^{\max }$ is the maximal hadronic energy allowed to be deposited in the cone of $R_{\gamma}$ 
around the photon. In the following we call this quantity hadronic or partonic leakage depending on whether the process is considered on the hadron or the parton level.

In a recent study [8] we proposed a simple way to approximate the isolated photon production that includes the direct photon contributions at the next-to-leading order (NLO) accuracy matched to shower Monte Carlo programs (SMCs). For the matching of NLO to SMC we use the POWHEG method [9, 10] as implemented in the POWHEG-BOX [11]. At previous EPSHEP conferences we reported $[12,13]$ about the PowHel framework that combines the POWHEG-BOX and the HELAC-NLO package [14] to simulate the hadroproduction of tt-pairs in association of other hard SM particles. The output of PowHel is simulated events stored according to the Les Houches accord [15] (LHE) that can be fed directly into SMC's to provide events at the hadron level. Using this framework we already provided events for several processes at the LHC [16, 17, 18, 19, 20, 21, 22].

The essence of our method to generate events with isolated hard photons is that we employ a loose smooth generation isolation (using Eq. (1) with small $\delta_{0}^{\text {gen }}$ ) to provide a pre-showered event sample. The events generated with a loose generation isolation contain only perturbative information, we neglect the non-perturbative (fragmentation) contribution. We however expect that in case of sufficiently loose generation isolation the fragmentation can be neglected within the expected uncertainty of matched NLO+PS predictions if the photon is harder than the accompanying jets. This statement is trivially true if the experimental isolation is a tighter version of the smooth isolation than that employed for event generation, while can be tested by comparison to data if a typical cone isolation is used. Thus our sample of LHEs can be considered sufficiently inclusive so that when a typical physical experimental isolation is employed the neglected fragmentation contribution is expected to be smaller than the renormalization and factorization scale uncertainties.

The technical details of our computations, using the example of isolated photon hadroproduction in association with a ț-pair, can be found in Ref. [8], where we concluded that the preshowered events reproduce the distributions at NLO accuracy within the expected precision. We have also shown that employing typical selection cuts (including the cone-type photon isolation the precise list is given in Ref. [8]), the physical predictions depend marginally on $\delta_{0}^{\text {gen }}$ at all stages of the event evolution if $\delta_{0}^{\text {gen }} \in[0.01,0.1]$. We show this independence of $\delta_{0}^{\text {gen }}$ of the cross section values after full SMC and given selection cuts are in Fig. 1 where the cross sections of the experimentally isolated photon are depicted as a function of the radius of experimental photon isolation cone, and of the hadronic leakage inside the photon isolation cone.

In order to estimate the size of the neglected fragmentation contribution one has to compare the predictions to experimental data which we present here for the case of massive vector boson + isolated photon production for which the ATLAS collaboration published results for both isolated photon +0 jet (exclusive) and isolated photon $+N(\geq 0)$ jets (inclusive) in the final state [23]. This final state has also been considered recently at NLO accuracy interfaced to a shower generator according to the POWHEG prescription supplemented with the MiNLO procedure [24]. In this work the fixed order result is matched to an interleaved QCD+QED parton shower, in such a way that the contribution arising from hadron fragmentation into photons is fully modeled. Thus for this process the comparison is possible not only for experimental results, but also with a theoretical prediction where the fragmentation is included through a shower model. We have implemented the event selection of ref. [23] and made predictions for the inclusive and for the exclusive case using events obtained with $\delta_{0}^{\text {gen }}=0.05$. In Fig. 2 we show our predictions for the transverse momen- 

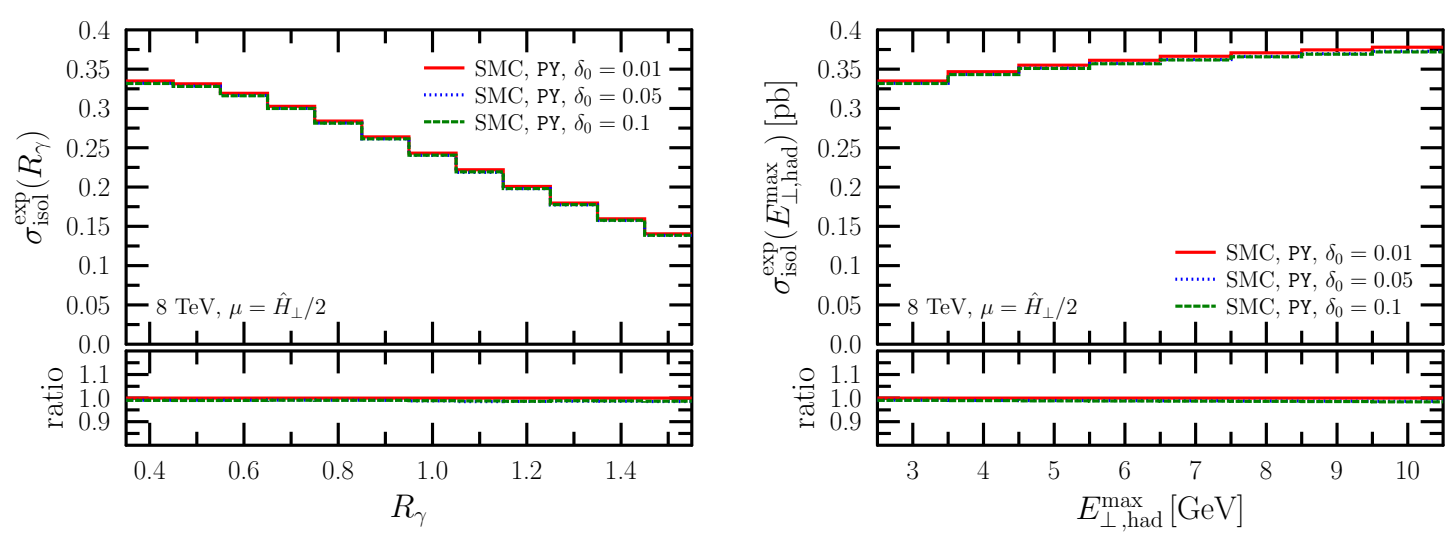

Figure 1: Isolated photon cross sections obtained after full SMC with different generation isolations using cuts listed in the text, as a function of the radius of experimental photon isolation cone (left), the hadronic leakage inside the photon isolation cone (right).

tum distribution of the isolated photon, compared to the predictions of ref. [24] and the results of ref. [23].
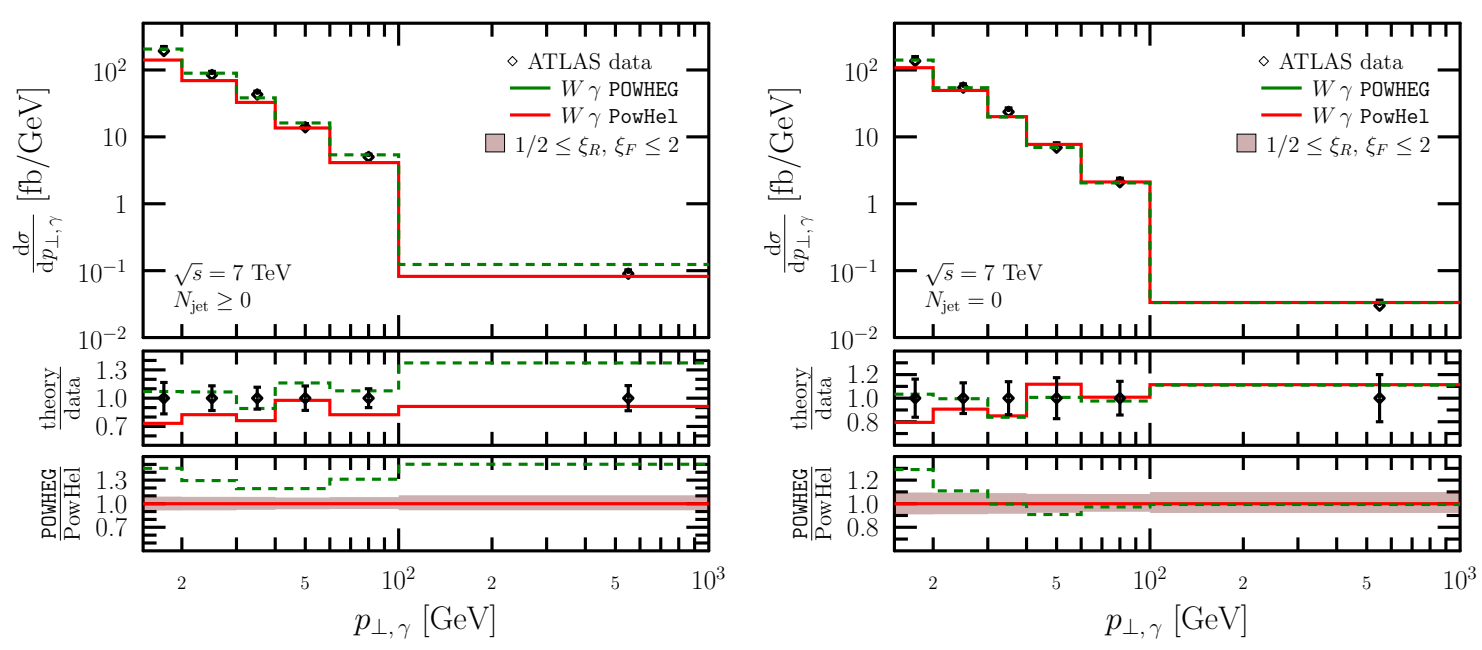

Figure 2: Distribution of the transverse momentum of the isolated photon for the $W+\gamma+N \geq 0$ jets (left) and $W+\gamma+0$ jet final states (right). The lower panels show the ratio of the predictions to the data.

We find that our approach gives a good description of the data if the radiated photon is harder than the accompanying jets. Thus for the exclusive case, the data overlap with our predictions for the full range within the uncertainty of our prediction. For this case the two theoretical predictions also coincide within the scale dependence band except for the first bin. For the inclusive case the two predictions differ and the difference, in principle, may be attributed to the neglected fragmentation contribution. However, it should be noted that the predictions of ref. [24] do not use non-perturbative information extracted from data, but a model of fragmentation. At the present accuracy of the data it is difficult to make a clear conclusion which prediction is favoured by experiment, but the following general trend seems to emerge: the harder the photon the better the agree- 
ment between our prediction and the data, while for the case of matched NLO to an interleaved QCD+QED parton shower, the agreement is better for small transverse momenta, with transition around $60 \mathrm{GeV}$ for the given selection cuts.

For studying the effect of the shower and hadronization as well as for simple phenomenology we chose PYTHIA-6.4.25 [25] to describe decay of heavy particles, QCD showering and hadronization. With the selection cuts in Ref. [8] we found small effect of the PS (in most of the phase space below $5 \%$ ). We also generated an event sample with $\delta_{0}^{\text {gen }}=0.01$ at $8 \mathrm{TeV}$ and fed into PYTHIA with the 2010 Perugia tune [26], omitting photon showers, making $\tau^{ \pm}$and $\pi^{0}$ stable and we turned off multi-particle interactions. For the phenomenology we used two default scales $\mu_{0}$ : (i) our default choice, the half the sum of transverse masses $\hat{H}_{\perp} / 2$, which was already motivated in [22] and (ii) the mass of the t-quark (the scale used in the first computation at NLO accuracy in Ref. [27]). To see the difference between the two scale choices a scale-uncertainty study is performed and scale-uncertainty bands are presented for the distributions obtained at the hadron level in Ref. [8]. We defined the renormalization and factorization scales as $\mu_{\mathrm{R}}=\xi_{R} \mu_{0}$ and $\mu_{\mathrm{F}}=\xi_{F} \mu_{0}$ and set the scale-dependence band as the upper- and lower-bounding envelopes of distributions taken with

$$
\left(\xi_{R}, \xi_{F}\right) \in\left\{\left(\frac{1}{2}, \frac{1}{2}\right),\left(\frac{1}{2}, 1\right),\left(1, \frac{1}{2}\right),(1,1),(1,2),(2,1),(2,2)\right\} .
$$

In general we found that the dynamical scale gave more uniform scale dependence of about $20 \%$ than the static one.

This method of generating events with a loose generation isolation of the photon is completely general and can be employed for other processes. In Ref. [28] we used it for making predictions for the associate production of two isolated hard photons with a t⿱t-t-pair at NLO accuracy matched to parton shower. With the default scale $\mu_{0}=\hat{H}_{\perp} / 2$, where the hat indicates that the underlying Born kinematics defines the scale $\hat{H}_{\perp}=m_{\perp, \mathrm{t}}+m_{\perp, \overline{\mathrm{t}}}+p_{\perp, \gamma_{1}}+p_{\perp, \gamma_{2}}$, used for both the renormalization and factorization scales, the NLO K-factor is 1.24 (for selection cuts given in Ref. [28]). The scaledependence of the cross section at LO and NLO accuracy is shown in Fig. 3. We see that varying the scale in the range $\left[\mu_{0} / 2,2 \mu_{0}\right]$ the scale uncertainty drops from $+30 \%-27 \%$ (at LO) to $+14 \%-$ $13 \%$ if NLO QCD corrections are included. It is interesting to note that choosing $\mu_{0}=\hat{H}_{\perp} / 4$ as default scale, the scale dependence remains the same, but the $\mathrm{K}$-factor decreases to $K \simeq 1.08$.

Using the predictions of Ref. [17] for $\mathrm{t} \overline{\mathrm{t}} H$ production at the hadron level, we can make predictions for both signal and irreducible background at the hadron level with the highest available precision. For the $\mathrm{t} \overline{\mathrm{t}}+\gamma \gamma$ background we take the events generated with $\delta_{0}=0.05$. For the signal we generated a new bunch of pre-showered events sharing the same parameters with the $t \bar{t}+\gamma \gamma$ sample, generated for the $13 \mathrm{TeV}$ LHC with CT10nlo PDF and accordingly chosen 2-loop $\alpha_{\mathrm{s}}$, $m_{\mathrm{t}}=172.5 \mathrm{GeV}, m_{H}=125 \mathrm{GeV}$. We chose the renormalization and factorization scales equal to each other and set to $\mu_{\mathrm{R}}=\mu_{\mathrm{F}}=m_{\mathrm{t}}+m_{H} / 2$. To make our predictions, we used PYTHIA -6.4 .25$ for simulating the evolution of the events to the hadron level, but with multiple interactions turned off.

The partonic final state contains a t-quark pair for both the signal and the background. To detect these t-quarks we used t-tagging as provided by the HEPTopTagger [29]. In order to perform t-tagging we followed the steps recorded in Ref. [28]. We show two sample distributions 


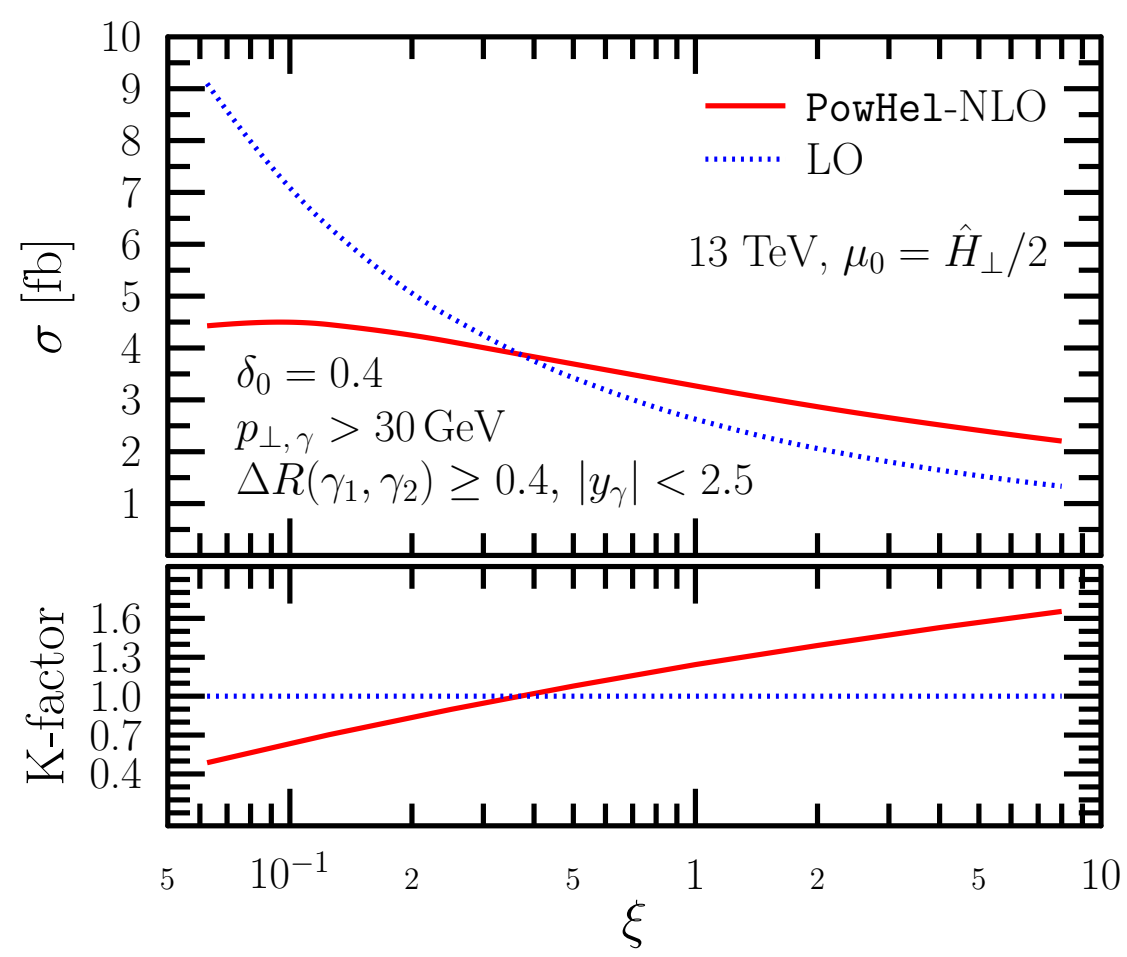

Figure 3: LO and NLO cross sections as functions of the scale variation parameter. Details of the setup and cuts applied can be found in the text.

obtained this way in Fig. 4: the invariant mass and the rapidity-azimuthal angle separation of the two-photon system. Clearly our selections are very strict as the cross section drops into the attobarn range, but also very effective: the Higgs boson the signal appears as a single, well-defined spike in the $m_{\gamma \gamma}$ spectrum with excellent signal/background ratio.

This is a part of a bigger project with the aim of providing event files produced by PowHel for processes $p p \rightarrow \mathrm{t} \overline{\mathrm{t}}+X$, where $X$ is a hard object (SM boson, jet, b-quark pair). These files are available at http://grid.kfki.hu/twiki/bin/view/DbTheory/ together with the corresponding version of the program. Using those events one can optimize the selection for $\mathrm{t} \overline{\mathrm{t}} H$-events.

\section{References}

[1] Aad G. et al., Phys.Lett. B, 710, 49 (2012).

[2] Chatrchyan S. et al., Phys.Lett. B, 710, 26 (2012).

[3] Beringer J. et al., Phys.Rev. D86, 010001 (2012).

[4] Maltoni F., Rainwater D. L. and Willenbrock S., Phys.Rev. D, 66, 034022 (2002).

[5] Belyaev A. and Reina L., JHEP, 0208, 041 (2002).

[6] S. Catani, M. Fontannaz and E. Pilon, Phys. Rev. D 58, 094025 (1998) [hep-ph/9803475].

[7] S. Frixione, Phys. Lett. B 429, 369 (1998) [hep-ph/9801442].

[8] A. Kardos and Z. Trócsányi, JHEP 1505, 090 (2015) [arXiv:1406.2324 [hep-ph]].

[9] P. Nason, JHEP 0411, 040 (2004) [arXiv:hep-ph/0409146].

[10] S. Frixione, P. Nason and C. Oleari, JHEP 0711, 070 (2007) [arXiv:0709.2092]. 

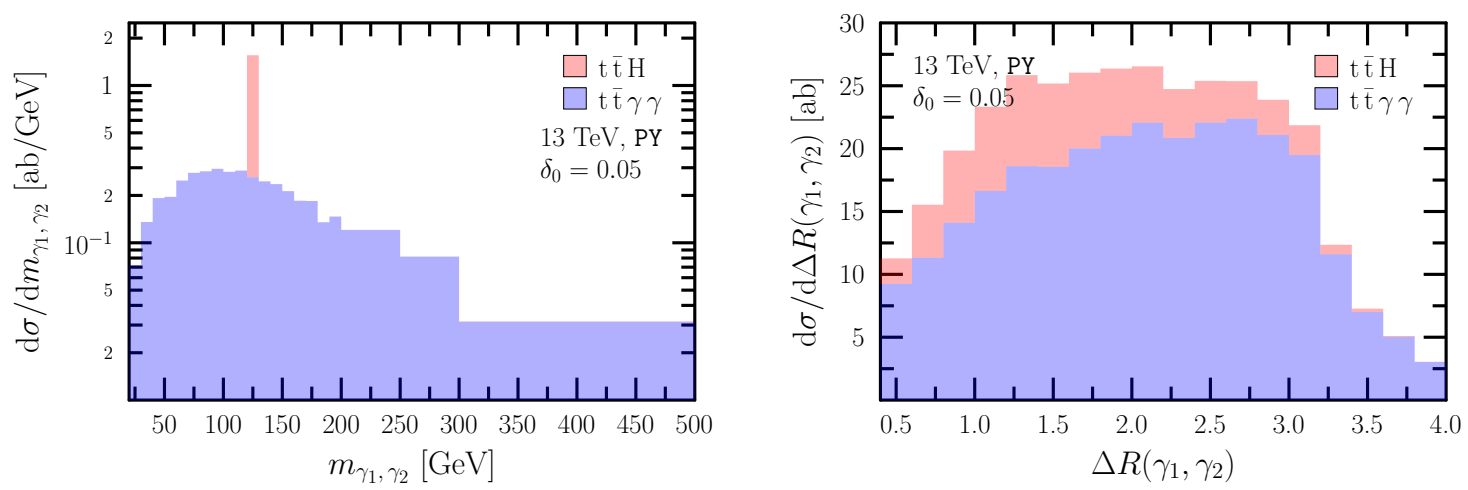

Figure 4: The invariant mass and rapidity azimuthal separation is shown for the diphoton system at the hadron level for the signal ( $\mathrm{t} \overline{\mathrm{t}} H$, red) and for the background $(\mathrm{t} \overline{\mathrm{t}}+\gamma \gamma$, blue) such that the signal sits on the top of the background. Further details of the calculation can be found in the main text.

[11] S. Alioli, P. Nason, C. Oleari and E. Re, JHEP 1006, 043 (2010) [arXiv:1002.2581].

[12] M. V. Garzelli, A. Kardos and Z. Trócsányi, PoS EPS -HEP2011, 282 (2011) [arXiv:1111.1446 [hep-ph]].

[13] M. V. Garzelli, A. Kardos and Z. Trócsányi, PoS EPS -HEP2013, 253 (2013).

[14] G. Bevilacqua, M. Czakon, M. V. Garzelli, A. van Hameren, A. Kardos, C. G. Papadopoulos, R. Pittau and M. Worek, Comput. Phys. Commun. 184, 986 (2013) [arXiv:1110.1499 [hep-ph]].

[15] J. Alwall et al., Comput. Phys. Commun. 176, 300 (2007) [arXiv:hep-ph/0609017].

[16] A. Kardos, C. Papadopoulos and Z. Trócsányi, Phys. Lett. B 705, 76 (2011) [arXiv:1101.2672 [hep-ph]].

[17] M. V. Garzelli, A. Kardos, C. G. Papadopoulos and Z. Trócsányi, Europhys. Lett. 96, 11001 (2011) [arXiv:1108.0387 [hep-ph]].

[18] M. V. Garzelli, A. Kardos, C. G. Papadopoulos and Z. Trócsányi, Phys. Rev. D 85, 074022 (2012) [arXiv:1111.1444 [hep-ph]].

[19] M. V. Garzelli, A. Kardos and Z. Trócsányi, PoS LL 2012, 057 (2012).

[20] M. V. Garzelli, A. Kardos, C. G. Papadopoulos and Z. Trócsányi, JHEP 1211, 056 (2012) [arXiv:1208.2665 [hep-ph]].

[21] M. V. Garzelli, A. Kardos, C. G. Papadopoulos and Z. Trócsányi, arXiv:1302.5381 [hep-ph].

[22] A. Kardos and Z. Trócsányi, arXiv:1303.6291 [hep-ph].

[23] G. Aad et al. [ATLAS Collaboration], Phys. Rev. D 87, no. 11, 112003 (2013) [Phys. Rev. D 91, no. 11, 119901 (2015)] [arXiv:1302.1283 [hep-ex]].

[24] L. Barze, M. Chiesa, G. Montagna, P. Nason, O. Nicrosini, F. Piccinini and V. Prosperi, JHEP 1412, 039 (2014) [arXiv:1408.5766 [hep-ph]].

[25] T. Sjostrand, S. Mrenna and P. Z. Skands, JHEP 0605, 026 (2006) [arXiv:hep-ph/0603175].

[26] P. Z. Skands, Phys. Rev. D 82, 074018 (2010) [arXiv:1005.3457 [hep-ph]].

[27]

[27] K. Melnikov, M. Schulze and A. Scharf, Phys. Rev. D 83, 074013 (2011) [arXiv:1102.1967 [hep-ph]].

[28] A. Kardos and Z. Trócsányi, Nucl. Phys. B 897, 717 (2015) [arXiv:1408.0278 [hep-ph]].

[29] T. Plehn, M. Spannowsky, M. Takeuchi and D. Zerwas, JHEP 1010, 078 (2010) [arXiv:1006.2833 [hep-ph]]. 